\title{
Scan to Pattern: How Body Scanning Can Help Transform Traditional Methods of Creating Pattern Blocks
}

\author{
Simeon GILL*, Yuting WANG,_Maryam AHMED, \\ Steven G. HAYES, Adrian R. G. HARWOOD, James GILL \\ The University of Manchester, Manchester, UK \\ DOI: $10.15221 / 18.236$ http://dx.doi.org/10.15221/18.236
}

\begin{abstract}
Body scanning provides one of the most efficient tools for recording information of the human body to support the development of body worn products. Traditionally the construction of garment patterns uses manual measurements and during the construction process applies some proportions, to create a pattern block [1], [2]. Traditional methods of drafting pattern blocks (slopers) apply very limited data from the body compared to the areas they cover and subsequently often require post drafting adjustments to achieve a suitable fit. Most pattern books have guidance on adjustments to blocks to accommodate figure variations [3]-[5]. These methods of block construction are well established and understood and have been used to inspire new approaches and propose theories for pattern block development [2], [6]. With advances in body scanning it is now possible to generate more measurements allowing for the body to have greater context in the process of pattern construction. This research retains the established $2 \mathrm{D}$ drafting methods and looks to explore further measurements than those traditionally used to create pattern blocks, these resulting blocks could then better reflect the individual variations in potential wearer size, shape and proportion. As well as looking to determine suitable measurements from a Size Stream (SS14) body scanner to inform the development of pattern blocks, this research tests an established skirt draft [4] using scan measurements, against a newly developed skirt drafting method which utilises the measurement capabilities of body scanning. The developed patterns are each tested on five dress forms. As well as assessing the resulting patterns, recommendations are made regarding how body scanning can be used to better inform pattern construction methods. This includes a contribution toward the theories of pattern construction, which will allow greater exploitation of body scanning technologies in developing better fitting and functioning garments. This research shows one means by which body scanning technologies can help to bridge the gap between traditional techniques of creating pattern blocks and the promising opportunities presented by body scanning technologies.
\end{abstract}

Keywords: 3D Body Scanning, 3D Virtual Simulation, Pattern Construction, Human Measurement, Size Stream

\section{Introduction}

Body scanning has the possibility to provide data about the body that can better inform the drafting of garment pattern blocks (Slopers). This research sought to address key areas which have not in existing research been looked at collectively. Firstly it sought to generate pattern blocks from body scan data, similar approaches have been taken by others [7], [8], it then applied these measurements in the creation of existing pattern blocks using established 2D drafting methods, others have used existing pattern block drafts [8]. The research then sought to use analysis of existing pattern making methods to support the creation of a new means to draft a pattern block, analysis of pattern drafts has been undertaken [9], [10], but in all current research there is little analysis of the approaches and theories of current pattern making practices or their history. Following from this pattern blocks for the skirt were generated and tested for fit with the results used to inform evolution of the new skirt draft method.

Taking this approach supported software development which allowed for the automated drafting of pattern blocks using body scan data. This provided the important step of reducing if not eliminating the use of proportions and set measurements in the draft process and proved the capability of body scanning in supporting pattern construction. During this process important questions were raised and key considerations identified for how body scanning analysis and software could be developed to support pattern drafting methods which better reflect the individual's size, shape and proportions. This research also raised key questions related to pattern theory (the relationship between the requirements of the pattern block with reference to the size, shape and proportion of the individual).

\footnotetext{
*simeon.gill.manchester.ac.uk
} 
The methods of Beazley and Bond [4] were selected for the skirt draft as they provide one of the few methods which is clear regarding ease levels incorporated into the pattern block. The skirt pattern was chosen specifically as it has one of the simplest structures as a pattern making method, depends on limited actual measurements, contains simple shaping and covers one of the key control section of the body [11].

\section{Background}

There has been long time recognition that body scanning can provide more data of the human body than traditional measurements alone [12]. Body scanning through the increased data has the propensity to support evolutions in pattern making methods, helping them to evolve past the theory poor opaque approaches currently available [11]. The opportunities to evolve pattern making methods are not new and work was undertaken to automated block drafting as long ago as the 1980's [13], though the work of Turner did not address key theories required to expand on the knowledge supporting the methods. However, unlike now, this approach did not have a suitable means to quickly capture body scanned data, which as shown in the work of Huang et al in 2010 and 2012 [7], [14] can be used to inform pattern making based on the body with recognition of its unique size, shape and proportion. Similar approaches to patternmaking based on scan data have been reported on by Tao and Bruniaux [8], here scan data is used to drive an existing method of pattern creation. Both these works show a recognition of the link between the body and the pattern, which is so important to evolve existing pattern making methods, though none address key considerations of how the drafting method works and any inbuilt weaknesses. Clear details of the relationship between the body and pattern are well documented in the work of Yang and Zhang [15]. There is still in spite of this research a number of important questions related to pattern making methods and their ability to respond to the uniqueness of the body [11].

An example of where more understanding of theory is required is when looking at the excellent work of Gu et al [16], here they map the crotch curve of the body as a series of offsets from the body. This work clearly displays the possibilities of using body scan data in informing pattern drafting. However this work suggests a direct relationship between the curve of the body and the curves of the pattern. If this proposal is contrasted to the analysis of the relationship between the scanned body and the pattern at the crotch undertaken by Mckinney et al [9], [17], it is clear that traditional trouser drafts create a crotch curve whose depth is smaller in width than the corresponding depth of the body. It seems evident when contrasting these two pieces of research that further consideration of historic practices are required to inform future developments in pattern making which seeks to harness new technology.

Published analysis of pattern making methods that are currently used have been undertaken by Gill and Chadwick [10], where analysis of bodice block drafts provided some guidance on ease levels within basic drafts. This research also indicated that patterns were influenced by the styles of the period when the methods developed, as can be seen in the curves to the CB bodice in the methods of Bray, a method developed when form fitting was desired. McKinney [17] also shows the importance of understanding practice when looking at the development of trouser patterns indicating the need for a deeper understanding of existing pattern making practices and better understanding of body to pattern relationships.

\section{Method}

A Size Stream SS 14 body scanner was used as the measurement tool to collect dimensions required for the pattern making. Initially measurements required for the Beazley and Bond [4] were mapped from the default measurements as had been undertaken with research into the bodice [1], when measurements were unavailable the custom measurement tool was used to create them. If a direct match measurement could not be determined, then a measurement was defined which would produce a body derived value as close to that specified in the draft process. One strength of Beazley and Bonds [4] methods is the clear descriptions of each required measurement accompanying the draft, this is further supported by reference to Beazleys excellent documenting of measurements required for pattern making [18].

The skirt block from Beazley and Bond (pg 30-32) [4] was selected as the control test draft and a new method was created based on taking some of the most successful elements from 17 existing pattern making methods. Each skirt method was analysed to determine the ease allowances incorporated at waist and hip, as well to guide on the dart placements and percentage suppression to shape the waist. 
The side seam uplift was also analysed, this is the amount added to the side seam to accommodate the anticipated extra length over the curve of the hip, compare to the CB and CF lengths.

Using extracted measurement from 5 scanned Alavaform dress forms representing a UK 10-12-14 range, a slim Asian figure and a UK size 24, skirt patterns were drafted manually for the Beazley method and for the new custom method. Similar to previous research [9], [10] each draft was checked for accuracy and then each pattern blocks was made up and fit tested on the dress forms.

Fit testing followed a process focused on key locations and ensured that each skirt met key criteria. The fit testing was undertaken independently by three experts using the same criteria to assess fit at key location and of key features. Using this approach it was possible to address key areas in the custom draft which required revision and to identify areas in the Beazley and Bond draft which might cause problems.

Following this the methods for development of the skirts were coded up as plots that can be automatically drafted within software fed by data from the body scanner. This required further testing and raised important questions related to pattern theory that would help further automate pattern construction for skirts and other block drafts.

\section{Results and Discussion}

Measurements required for pattern making. During the analysis of the pattern making methods and in determination of measurements it was clear that detailed descriptions of both manual measurements required for the draft and detailed descriptions of scan measurements were essential to correctly apply extracted scan data in existing methods. Even using the Size Stream custom measurement tool some measurements which were required, or identified as important could not be created. It is recommended consideration be given to data required for pattern making as a basic output of all scan systems to allow them to integrated into existing approaches to pattern making.. In some instances it was possible to define a measurement that could be used, but might not follow directly the contour of the outer body. It would be useful to be able to create custom surface lengths between key landmarks that would better describe the body and inform the pattern. An example would be in determining the waist to hip lengths. Beazley and Bond [4] suggest measurements between the waist and hip at the side seams and the CF and $\mathrm{CB}$ to create the corresponding depths in the pattern draft. These measurements would be extremely useful in the new custom skirt draft as well and give a specific example further highlighting the need to align body scanning measurements to existing pattern applications. Something which is evident when looking at body to pattern relationships that are proposed in existing research [14], [15].

Measurements used in creating the pattern drafts were not always taken on the forms by the body scanner at the corresponding locations where they were marked on the form. This meant during the fitting stage the heights of the waist measurements needed to be marked onto the forms to ensure the toiles were correctly matched. This highlights the potential difference between the scan defined measurement locations and the marked locations on dress forms and suggests a clear need to consider this in research.

Within the fit testing key areas of the pattern were considered, including fit at the waist and side seam, the balance of the hem, ease at waist and hip, as well as the positioning, length and size of the darts (Table 1). These were considered to be important locations to assess in fitting a skirt.

Table 1: Assessment of skirt toiles from patterns

\begin{tabular}{|c|c|}
\hline \multicolumn{2}{|l|}{ Skirt Toile Assessment Summary } \\
\hline Method = Gill 2018 & Method = Beazley \& Bond 2003 \\
\hline $\begin{array}{l}\text { Waist - Skirt waists sit up about } 1.5 \mathrm{~cm} \text { from tape } \\
\text { marked waist on form }\end{array}$ & $\begin{array}{l}\text { Waist - Skirt waists sit up about } 1.5 \mathrm{~cm} \text { from tape } \\
\text { marked waist on form. }\end{array}$ \\
\hline Hip Shape on SS - Requires fitting & Hip Shape on SS - Requires fitting \\
\hline Ease - ease is ok in most cases & Ease - waists are too loose \\
\hline Fr Dart - ok & Fr Dart - ok \\
\hline $\begin{array}{c}\text { Bk Dart - Positions are ok, but lengths need to be } \\
\text { shortened about } 4 \mathrm{~cm}\end{array}$ & $\begin{array}{l}\text { Bk Dart - Positions are ok, but lengths need to be } \\
\text { lengthened. }\end{array}$ \\
\hline Balance - SS slightly away toward the front & Balance - ok \\
\hline
\end{tabular}


As these pattern drafts were both driven by body scan measurements, the position of the waist across the five dress forms was approximately $1.5 \mathrm{~cm}$ higher that the marked waist on the form. The hip shape on both drafts required further fitting to correctly contour to the shape, it must be noted that current drafts for skirts shape the waist without guidance from the body between the waist and hip and having some further measurements of this area would help create suitable shaping here. There are also considerations related to the shaping as the sideseam could be viewed as a shaped dart and as such requires balancing in dart leg length for it to sit correctly, this raise issues about how the waist circumference is divided, if it is done within the draft as in Beazley, or using a division set within the scanner as in Gill. Ease in the method by Gill was judged to be okay in the experience of the assessors, whilst Beazley and Bonds method appeared to incorporate too much ease, at the waist this could be due to the expectation the skirt waist would be eased onto the waistband.

Interestingly the back darts on Beazley and Bond were judged to be too short, whilst those on the method by Gill, which were guided by depths of the scanner needed to be shortened in the back. It is evident the dart requirements relate to the curve of the buttocks and suggest some indication as to theories which can be developed to guide on dart length for the back of a garment on using body scanned data. The Beazley and Bond method like most skirt pattern making methods uses a fixed length for the back darts, Gill applied a method based on the seat, taken at the greatest buttock prominence.

Neither pattern making method created a block which fitted correctly first time, however the method by Gill using body scan data provided the greatest opportunity to correct the block in future devolvement and being driven more directly by the body scan provided a direct opportunity to use data from the body to improve its fit and future development of the method.

\section{Conclusions}

Body scanning provides a very effective tool for determining dimensions of the body which can be used to evolve pattern making methods. Whilst further development of measurements is required to fully explore this potential, it is clear that scanning enables a more informed set of data that can help build pattern making theory, which in turn will support efforts at informed automation of pattern making. It is vital to support the evolution of pattern making that efforts be made to create systems of analysis which take all measurements required for existing established pattern making methods, this will help systems integrate into existing approaches used in industry and academia.

These types of studies raise important areas of study in understanding pattern theory and how the pattern shape is created in relation to an individual's size, shape and proportion. There remain key questions about what influences the position of the waist darts in the skirt, though in this study the front dart seems aligned to the abdomen projection at the front and the buttocks at the back. Further development of analysis tools could help to provide a clearer means to determine the suitable dart lengths whilst a more thorough analysis of arcs, depths and widths would help to guide on dart openings.

Body scanning provides an efficient tool to create data to support pattern making and can if programmed correctly help evolve the practice of making blocks for both mass production and made to measure.

\section{References}

[1] S. Gill, M. Ahmed, C. J. Parker, and S. G. Hayes, "Not All Body Scanning Measurements Are Valid: Perspectives from Pattern Practice," in 8th International Conference and Exhibition on 3D Body Scanning and Processing Technologies, 2017.

[2] S. Gill, "A review of research and innovation in garment sizing, prototyping and fitting," Text. Prog., vol. 47, no. January, pp. 1-85, 2015.

[3] W. Aldrich, Metric Pattern Cutting for Women's Wear, 6th ed. Chichester, UK: Wiley, 2015.

[4] A. Beazley and T. Bond, Computer-aided pattern design and product development. Oxford: Blackwell Science, 2003.

[5] H. J. Armstrong, Pattern Making for Fashion Design, 5th ed. Upper Saddle River, N.J.: Pearson/Prentice Hall, 2010. 
[6] E. C. Hlaing, S. Krzywinski, and H. Roedel, "Garment prototyping based on scalable virtual female bodies," Int. J. Cloth. Sci. Technol., vol. 25, no. 3, pp. 184-197, 2013.

[7] H. Huang, P. Y. Mok, Y. L. Kwok, and J. S. Au, "Automatic Block Pattern Generation from a 3D Unstructured Point Cloud," Res. J. Text. Appar., vol. 14, no. 1, pp. 26-37, 2010.

[8] X. Tao and P. Bruniaux, "toward advanced three-dimensional modeling of garment prototype from draping technique," Int. J. Cloth. Sci. Technol., vol. 25, no. 4, pp. 266-283, 2013.

[9] E. McKinney, S. Gill, A. Dorie, and S. Roth, "Body-to-Pattern Relationships in Womens Trouser Drafting Methods: Implications for Apparel Mass Customization," Cloth. Text. Res. J., vol. 35, no. 1, pp. 16-32, 2017.

[10] S. Gill and N. Chadwick, "Determination of ease allowances included in pattern construction methods," Int. J. Fash. Des. Technol. Educ., vol. 2, no. 1, pp. 23-31, Mar. 2009.

[11] S. Gill, "A review of research and innovation in garment sizing, prototyping and fitting," Text. Prog., vol. 47, no. 1, 2015.

[12] E. Bye, K. L. Labat, and M. R. Delong, "Analysis of Body Measurement Systems for Apparel," Cloth. Text. Res. J., vol. 24, no. 2, pp. 66-79, Mar. 2006.

[13] J. P. Turner, "A Computerised Technique for the Production of Clothing Patterns," PhD Thesis, The University of Manchester, 1986.

[14] H. Q. Huang, P. Y. Mok, Y. L. Kwok, and J. S. Au, "Block pattern generation: From parameterizing human bodies to fit feature-aligned and flattenable 3D garments," Comput. Ind., vol. 63, no. 7, pp. 680-691, 2012.

[15] Y. Yang and W. Zhang, "Prototype garment pattern flattening based on individual 3D virtual dummy," Int. J. Cloth. Sci. Technol., vol. 19, no. 5, pp. 334-348, 2007.

[16] B. Gu, W. Lin, J. Su, and B. Xu, "Predicting Distance Ease Distributions on Crotch Curves of Customized Female Pants," Int. J. Cloth. Sci. Technol., vol. 29, no. 1, p. Early Cite Article, 2017.

[17] E. C. McKinney, E. Bye, and K. LaBat, "Building patternmaking theory: a case study of published patternmaking practices for pants," Int. J. Fash. Des. Technol. Educ., vol. 5, no. 3, pp. 153-167, 2012.

[18] A. Beazley, "Size and fit: Procedures in undertaking a survey of body measurements - Part 1," J. Fash. Mark. Manag., vol. 2, no. 1, pp. 55-85, 1997. 